\title{
3D Markov Random Fields and Region Growing for Interactive Segmentation of MR Data
}

\author{
Marc Liévin, Nils Hanssen, Peter Zerfass, and Erwin Keeve \\ Surgical Simulation and Navigation Group \\ Research center c a e s a $\mathrm{r}$ \\ Friedensplatz 16, 53111, Bonn, Germany \\ http://www.caesar.de/ssn
}

\section{Introduction}

Segmenting medical structures is mandatory in any computer assisted surgery system. This major field must be addressed in order to build realistic and accurate 3D models of patient individual anatomical structures. Magnetic Resonance Imaging (MRI) is becoming part of daily routine in clinical work. Whereas scanning speed and slice numbers increase each year, segmenting such data is still a challenging problem. Moreover, the segmentation stage remains time limiting in pre-operative planning and intra-operative guidance. Indeed, interactive tools, like live wire or intensity-based thresholding, requires a pre or post-filtering to homogenize areas. Common medical filters, such as median or morphology-based, are actually non adapted for MR noise removal. Their main side effect is to remove boundaries when applied on Gaussian corrupted data. Next, numerous steps spend efforts in reconstructing lost information and current approaches are therefore non interactive.

\section{Methods}

Our method mainly differs from the literature by grouping time-consuming steps in the first stage. In a multi-stage process, the framework uses statistical clustering as a preprocessing stage before region growing. To reduce the complexity of the filtering and to increase the interactivity with the user, the initial raw data is quantized in a sufficient but limited number of clusters (e.g. 16 quantization steps).

Next, a filtering based on Markov random fields (Mrfs) clustering is applied on the whole volume. The main purpose of the Mrfs is to link observations from data and relevant labels in a smart way. Considering our clustering as a non-linear filter, the observations are the raw data itself and our label set the corresponding values after quantization. The originality of our procedure is the definition of the metric neighborhood structure, which may contained from 6 to 26 neighbors in a 3D metric space, depending on how far accuracy is needed. The metric potential function is defined as the inverse of the Euclidian distance in millimeters between two neighbors. Next, less than 10 iterations on the field are enough to obtain homogeneous fields respecting boundaries and initial data value range. 
Region growing uses connectivity and homogeneity properties to link neighbors. The growth starts from one or several seed points located interactively by the user in the slice views. The homogeneity criterion is based on statistical distribution of the filtered values. The user interactively moves the threshold from low values to higher values until the result is acceptable.

\section{Results and Discussion}

Accuracy and relevance of our approach are validated on a high-resolution MR study of a male knee, with 512x512x120 images and a voxel size of $0.27 \times 0.27 \times 1.0 \mathrm{~mm}$ (50 Mbytes data set). Figure 1 presents the result of the 3D Mrfs filtering with 16 clusters of a whole slice. On RISC $400 \mathrm{MHz}$ architecture, the processing takes only between 0.5 and $1 \mathrm{sec}$. per slice, e.g. up to 5-10 $\mathrm{min}$. for the whole volume. Next, the user may interact during the region growing process with an update rate under the second, 0.2 sec. for cartilage growth over 138000 voxels.



Fig. 1. Mrfs filtering and region growing results on the Femural Cartilage area (Transversal View; In white, the ROI) a) MR slice filtered with 16 clusters; b) Zoomed Views of the ROI: 1) The raw data, 2) The Mrfs filtered data, 3) The Mrfs filtered data with superimposed in white region growing result of the cartilage.

The filtering algorithm only requires two tuning steps from the user: the neighborhood system (6 or 26 neighbors) and the quantization step. Integrated in a Vtk class (Kitware Inc.), this filter may be easily added to any medical pipeline. Extracting the 3D shape of challenging areas, such as cartilage in the knee, becomes easily and quickly feasible. We need now to extend this approach with new interactive tools like live wire or gradient methods. Integrating these procedures with volume rendering for advanced interactive segmentation is also under study.

\section{Acknowledgment}

We thank Ron Kikinis and the Surgical Planning Laboratory at Brigham and Women's Hospital Harvard Medical School, for making available the MR and manual segmented dataset of the knee. 